\title{
Singlet fermions on curved extra dimension tori
}

\author{
I. Vilja* \\ Department of Physics, FIN-20014 University of Turku, Finland
}

December 18, 2018

\begin{abstract}
A model with two curved compact extra dimensions is introduced. The model is based on a four-brane immersed in a six dimensional space, where the extra dimensions are compact but not flat. They have topology of torus. The form of metric in the empty bulk is studied and the gauge singlet fermion structure showed to be very simple. It contain only one massless low energy mode which couples to brane matter, while the massive modes are not related to the volume of torus while the Planck mass is related to the volume of extra dimensions. In the model "our" brane is situated on a nearly singular line of the torus.
\end{abstract}

*vilja@utu.fi 


\section{Introduction}

The intriguing idea, that the physical space may have more that three dimensions has got a lot attention recently. The idea itself dates bect to the beginning of the 20th century, to the works of Nordstöm, Kaluza and Klein [1]. They attempted to unify gravity and electromagnetism by introducing a fifth dimension. The idea reappeared in string theories, where unification all four known fundamental interactions was made in ten or eleven dimensional space-time. Originally the string theorists supposed, that the left-over dimensions are compactified to extremely short distances, which therefore would have only a little (direct) impact to low energy physics and particle phenomenology. Later, however, it was found that these extra dimensions, or some of them, can be relatively large, lie on an intermediate scale [2]. This finding is related to the uncertainty of the measurements of gravitational forces. They indicate, that the extra dimensions may be even as large as $\sim 1 \mathrm{~mm} \mathrm{[3,} \mathrm{4,} \mathrm{5].} \mathrm{These}$ considerations have boosted a new wave of research on large extra dimensions because the may have testable consequences in present day or near future experiments.

In the core of this research is the notion, that the Planck scale $M_{P l}$ is possibly not the "true" gravity scale, but only an effective gravity scale related to our four dimensional spacetime $M_{4}$ of our natural perception [6, 7, 8]. The gravity scale $M_{*}$ of the space-time which includes $M_{4}$ and $n$ the large extra dimensions, is related to Planck scale by a well defined equation $M_{P l} \sim M_{*}^{n+2} V^{(n)}$, where $V^{(n)}$ is an extra dimension size related factor. Depending on the form of $V^{(n)}, M_{*}$ may be considerably lower scale than $M_{P l}$. Moreover, the particles of the Standard model (SM) or other gauge symmetries are supposed to live only on a four dimensional slices of all large dimensions (e.g. on $M_{4}$ ) [7]. This slices, called the branes, may have at least two possible realizations. They may be defined by solitonic configurations of underlying string theory, when they have finite but small width being effectively four dimensional. Or they may be constructed as a singular point of an orbifold, when they are real four dimensional manifolds [9].

In these large extra dimension models the particles which are gauge singlets may propagate outside the brane(s), in so called bulk [7], forming bulk matter. Interactions between the brane and bulk matter may therefore rise interesting consequences. Righthanded neutrinos may be this kind singlet fermions [10, 11]. This would explain quite naturally e.g. the lightness of neutrinos: their masses would be suppressed by factor $\sim M_{*} / M_{P l}$. A lot of work on particle phenomenology of extra dimension models has done during last years [12, 13]. Also the cosmological implications of the extra dimensions have been subject of numerous 
studies [9, 14].

In practise, there is two fundamental types of extra dimension constructions. One may have compact extra dimensions, so called ADD-type models [ 6], or the extra dimensions may be non-compact but "wrapped", when one speaks of RS-models [15]. In ADD-models the factor $V^{(n)}$ is simply the volume of the extra dimensions, while it in RS-models is merely related to the normalization of gravitons. In either case the particles living in the bulk shows up to form to an infinite Kaluza-Klein tower of excitations when observed on the brane.

In the present paper we study a model which lies somewhere in-between ADD-models and RS-models. The manifold of the extra dimensions is a compact one, and the bulk empty. We study a four-brane embedded on a six dimensional space of large dimensions. However we do not suppose it to be a flat manifold but allow it be curved, somehow wrapped like in RS-models. This makes possible to obtain a considerablysimple spectrum of excitations of the bulk matter, in particular bulk fermions as the right handed neutrinos. We also study the general features of this model and propose a more detailed, simple construction.

\section{Geometry on a torus}

We coordinatise the six dimensional space with $z^{M}$, where $M=0, \ldots, 5$, where the first four coordinates are associated to the Minkowski space. The two extra dimensions have topology of two-torus $\mathrm{T}^{2}$ with coordinates $y^{a}, a=1,2$. The metric of these extra dimensions, $d s_{E}^{2}=g_{a b} d y^{a} d y^{b}$, can quite generally be written in the form $\left(y^{1}=\phi, y^{2}=\theta\right)$

$$
d s_{E}^{2}=a^{2} d \phi^{2}+f(\phi, \theta)^{2} d \theta^{2},
$$

applying suitable coordinate transformations. Here we can assume, that $a$ is a constant and the range of the angle variables $\phi$ and $\theta$ is the full circle. Because of a stationary situation, the metric of extra dimensions do not depend on Minkowski space coordinates, in particular on time: Riemann and Ricci curvature tensors do not mix Minkowski and extra dimension components. Thus the only non trivial components are $R_{\theta \phi \theta \phi}=-f f_{\phi \phi}, R_{\theta \theta}=f f_{\phi \phi} / a^{2}$ and $R_{\phi \phi}=f_{\phi \phi} / f$ and hence the curvature scalar of the extra dimensions reads $\mathcal{R}=-2 f_{\phi \phi} /\left(a^{2} f\right)$. The volume of the torus can be also calculated, giving naturally

$$
V_{2}=a \int_{0}^{2 \pi} d \theta \int_{0}^{2 \pi} d \phi f(\phi, \theta) .
$$

The Einstein tensor $E_{\nu}^{\mu}=R_{\nu}^{\mu}-\frac{1}{2} g_{\nu}^{\mu} \mathcal{R}$ reads now $E_{\nu}^{\mu}=-\delta_{\nu}^{\mu} \mathcal{R}$ while extra dimension components are equal to zero: $E_{b}^{a}=E_{\mu}^{a}=0$. The six dimensional Einstein equation

$$
E_{B}^{A}=8 \pi G_{6} T_{B}^{A}
$$


implies thus, that the bulk is empty and possible branes are located in $\phi$-direction at points $\phi_{i}$. Eq. (3) means that one has indeed a four branes instead of a three-branes: the position of brane is not fixed in $\theta$-dimension, but includes it as whole. Thus the geometry describes empty bulk with branes of tension $\sigma_{i}$ located given points. The piecewise defined bulk solution, the scale function $f$ consists of simple positive linear pieces

$$
f(\phi, \theta)=f_{i}(\theta)+\left[f_{i+1}(\theta)-f_{i}(\theta)\right] \frac{\phi-\phi_{i}}{\phi_{i+1}-\phi_{i}}, \quad \phi_{i} \leq \phi<\phi_{i+1}
$$

where, without loss of generality can be chosen $\phi_{1}=0, \phi_{K+1}=2 \pi$ representing the same point. Note, that due to periodicity the number of branes is 0 or larger than 1 . Naturally, if it equals zero, $f$ is a function of $\theta$ only and this function can be absorbed to the coordinate itself. The positions of branes, functions $f_{i}$ and the brane tensions $\sigma_{i}$ are related by crossing rules, the jump conditions, which reads as

$$
\frac{\Delta f_{i}}{\Delta \phi_{i}}-\frac{\Delta f_{i-1}}{\Delta \phi_{i-1}}=-4 \pi M_{*}^{-4} \sigma_{i} a^{2} f_{i}
$$

where $\Delta f_{i}=f_{i+1}-f_{i}$ and $\Delta \phi_{i}=\phi_{i+1}-\phi_{i}$ and $M_{*}$ is the six-dimensional fundamental gravity scale related to six-dimensional gravity constant by $G_{6}=M_{*}^{-4}$. With this notation The volume of extra dimensions reads

$$
V_{2}=a \int_{0}^{2 \pi} d \theta \sum_{i} \Delta \phi_{i} \frac{f_{i+1}+f_{i}}{2}
$$

and relation between the four-dimensional gravitational scale (the Planck scale $M_{P l}$ ) and sixdimensional scale $M_{*}$ reads now as $M_{P l}^{2}=M_{*}^{4} \int d^{2} y \sqrt{-g_{6}}=V_{2} M_{*}^{4}$. Of course, if functions $f_{i}$ are independent on coordinate $\theta$, the integration contributes only with factor $2 \pi$. Moreover, if there are also only two branes, the jump conditions are simply,

$$
\Delta f\left(\frac{1}{2 \pi-\phi^{\prime}}+\frac{1}{\phi^{\prime}}\right)=-16 \pi G_{6} a^{2} \sigma_{1} f_{1}=16 \pi G_{6} a^{2} \sigma_{2} f_{2},
$$

and the volume reads

$$
V_{2}=2 \pi^{2} a\left(f_{1}+f_{2}\right)
$$

where $\Delta f=f_{2}-f_{1}$, and $\phi^{\prime} \neq 0$ is the position of the other brane. Should be again emphasized, that one single brane is not possible because it leads to constant $f$ at whole bulk: it implies no discontinuities, no tension and no matter.

Because our aim is to calculate the equations of motion for fermion fields, we need the spin connection defined by

$$
\Omega_{M}=\frac{1}{2} \Gamma^{A B} V_{A}^{N} \partial_{M} V_{B N}
$$


where $V_{A}^{N}$ is the moving frame (sixbein) $V_{A}^{N}=\operatorname{diag}(1,1,1,1, a, f), \Gamma^{A B}=\frac{i}{2}\left[\Gamma^{A}, \Gamma^{B}\right]$ and matrices $\Gamma^{A}$ are the $8 \times 8$ gamma matrices for six dimensional space. In this presentation we use the gamma matrices

$$
\Gamma^{\mu}=\left(\begin{array}{cc}
0 & \gamma^{\mu} \\
\gamma^{\mu} & 0
\end{array}\right), \quad \Gamma^{4}=\left(\begin{array}{cc}
0 & 1_{4} \\
-1_{4} & 0
\end{array}\right), \quad \Gamma^{5}=-i\left(\begin{array}{cc}
0 & \gamma^{5} \\
\gamma^{5} & 0
\end{array}\right)
$$

where $\gamma^{\mu}$ 's are the usual four-dimensional gamma matrices. For the 2-torus metric (11), however, all components of the spin connection vanish. Thus the covariant derivative for spin $\frac{1}{2}$-field reads simply $\mathcal{D}_{A}=V_{A}^{M} \partial_{M}$. Note also, that the six-dimensional chirality operators are

now given by $P_{ \pm}=\frac{1}{2}\left(I_{8} \pm \Gamma^{7}\right)$, where $\Gamma^{7}=-\Gamma^{0} \Gamma^{1} \cdots \Gamma^{5}=\operatorname{diag}\left(1_{4},-1_{4}\right)$ is the generalization of $\gamma^{5}$ in six dimensions.

If the dimension coordinated by $\theta$ is a maximally symmetric subspace, one can even choose a coordinate system where $f$ in independent of $\theta$-coordinate itself. It is naturally equivalent with the assumption, that the function $f$ can be split to product of separate $\theta$ and $\phi$ dependent parts.

\section{Singlet fermions on a general torus}

In particle theory models the gauge-singlet fermions, which are thus not confined on a brane, live on the whole six-dimensional space. The most natural such particles are the singlet neutrinos, which may appear even in the simplest extensions of the standard model. To study their properties on the manifold $M_{4} \times T^{2}$ we have to write down the action for the singlet neutrinos. The free field Lagrangian reads as

$$
S_{N}=\int d^{4} x d^{2} z V\left[i \bar{N} \Gamma^{A} \mathcal{D}_{A} N+\text { h.c. }-M \bar{N} N\right]
$$

where $V=\operatorname{det}\left(V_{A}^{N}\right)=a f, N$ is the fermion field at six dimensions, $\bar{N}=N^{\dagger} \Gamma^{0}$ and $M$ is the mass of the fermion field. We also denote chiral six dimensional eight component fermions as $N_{ \pm}=P_{ \pm} N$ and identify them with four component chiral fermions, i.e.

$$
N=\left(\begin{array}{c}
N_{+} \\
N_{-}
\end{array}\right)
$$

Note, that $N_{ \pm}$are identifiable to four dimensional Dirac fermions. Using these components, the action reads

$$
\begin{aligned}
S_{N}= & \int d^{4} x d^{2} z V\left[i\left(\bar{N}_{-} \bar{N}_{+}\right)\left(\begin{array}{cc}
0 & 1 \\
1 & 0
\end{array}\right) \otimes \gamma^{\mu} \partial_{\mu}\left(\begin{array}{c}
N_{+} \\
N_{-}
\end{array}\right)\right. \\
& +\frac{i}{2}\left(\bar{N}_{-} \bar{N}_{+}\right)\left(\begin{array}{cc}
0 & 1 \\
-1 & 0
\end{array}\right) \otimes \mathbb{1} \frac{1}{a} \partial_{\phi}\left(\begin{array}{c}
N_{+} \\
N_{-}
\end{array}\right)+\text {h.c. }
\end{aligned}
$$




$$
\begin{aligned}
& +\frac{i}{2}\left(\bar{N}_{-} \bar{N}_{+}\right)\left(\begin{array}{cc}
0 & \gamma^{5} \\
\gamma^{5} & 0
\end{array}\right) \otimes \mathbb{1} \frac{-i}{f} \partial_{\theta}\left(\begin{array}{c}
N_{+} \\
N_{-}
\end{array}\right)+\text {h.c. } \\
& \left.+M\left(\bar{N}_{-} \bar{N}_{+}\right)\left(\begin{array}{c}
N_{+} \\
N_{-}
\end{array}\right)\right]
\end{aligned}
$$

where now $\bar{N}_{ \pm}=N_{ \pm}^{\dagger} \gamma^{0}$. The corresponding equation of motion reads now

$$
-i \gamma^{\mu} \partial_{\mu}\left(\begin{array}{c}
N_{+} \\
N_{-}
\end{array}\right)=\frac{i}{a} \partial_{\phi}\left(\begin{array}{c}
-N_{+} \\
N_{-}
\end{array}\right)+i \gamma^{5} \frac{-i}{f} \partial_{\theta}\left(\begin{array}{c}
N_{+} \\
N_{-}
\end{array}\right)+\frac{i}{2 a} \frac{\partial_{\phi} f}{f}\left(\begin{array}{c}
-N_{+} \\
N_{-}
\end{array}\right)+M\left(\begin{array}{c}
N_{-} \\
N_{+}
\end{array}\right) .
$$

This equation can be solved by separating Minkowski space and torus variables leading to tedious calculations, where the components mix strongly. Therefore, we henceforth assume that the six-dimensional field $N$ is chiral field in the sense of six-dimensional theory, e.g. $N_{-}=0$. This corresponds the assumption $M=0$, which decouple the six dimensional chiral fields. It should be also noted, that for chiral six-dimensional spinors there are no separate Majorana-couplings either, because Majorana fermions at six dimensions are chiral themselves.

Hence we may rewrite the equation of motion for $N_{+}$field alone as

$$
-i \gamma^{\mu} \partial_{\mu} N_{+}=-\frac{i}{a} \partial_{\phi} N_{+}-\frac{i}{2 a}\left(\partial_{\phi} \ln f\right) N_{+}+\frac{1}{f} \gamma^{5} \partial_{\theta} N_{+}
$$

In this equation we perform a generalized Fourier series expansion: The right side of eq. (15) is set equal to $-M_{+} N_{+}$, where $M_{+}=m_{+}+i \mu_{+} \gamma^{5}$ and $m_{+}$and $\mu_{+}$are real numbers. Then we write the field as a sum of its chiral components $N_{+}=N_{++}+N_{+-}$with $\gamma^{5} N_{+ \pm}= \pm N_{+ \pm}$, so that the equation of motion for the fields reads as

$$
\begin{aligned}
-\frac{i}{a} \partial_{\phi} N_{++}+\frac{1}{f} \partial_{\theta} N_{++}-\frac{i}{2 a}\left(\partial_{\phi} \ln f\right) N_{++} & =-M_{+} N_{++}, \\
-\frac{i}{a} \partial_{\phi} N_{+-}-\frac{1}{f} \partial_{\theta} N_{+-}-\frac{i}{2 a}\left(\partial_{\phi} \ln f\right) N_{+-} & =-M_{+} N_{+-} .
\end{aligned}
$$

By writing the chiral fields as $N_{+ \pm}=H_{ \pm}(\phi, \theta) \psi_{ \pm}(x)$, where $\psi_{ \pm}$are usual four dimensional fermion fields, one finds, that the equations of motion for $H_{ \pm}$are

$$
-\frac{i}{a} \partial_{\phi} H_{ \pm} \pm \frac{1}{f} \partial_{\theta} H_{ \pm}-\frac{i}{2 a}\left(\partial_{\phi} \ln f\right) H_{ \pm}=-\left(m_{+} \pm i \mu_{+}\right) H_{ \pm}
$$

where upper/lower signs belong to same equation. Immediately one sees, that

$$
H_{ \pm}(\theta, \phi)=e^{i \nu_{ \pm} \theta} K_{ \pm}(\phi)
$$

where $\nu_{ \pm}$are due to $\theta$-periodicity integers and $K_{ \pm}$are functions of $\phi$ only. Inserting (18) to (17), one finds solution

$$
K_{ \pm}(\phi)=\exp \left[i a \int_{0}^{\phi} d u\left(-m_{+}+\frac{i}{2 a} \partial_{u} \ln f(u) \pm i\left(-\mu_{+}-\frac{\nu_{ \pm}}{f(u)}\right)\right)\right] K_{ \pm}(0) .
$$


Again, consistency with periodicity requires, that $K_{ \pm}(2 \pi)=K_{ \pm}(0)$ and we obtain relation for mass parameters $m_{+}$and $\mu_{+}$:

$$
-a m_{+}=n, \quad n \in \mathbb{Z}
$$

and

$$
\int_{0}^{2 \pi} d \phi\left(\mu_{+}+\frac{\nu_{ \pm}}{f(\phi)}\right)=0 .
$$

Note, that $\int_{0}^{2 \pi} d u \partial_{\phi} \ln f(u)=f(2 \pi)-f(0)$ vanish automatically due to periodicity of $f$. Moreover, Eq. (21) should hold for both $\nu_{+}$and $\nu_{-}$. Thus we find that they have to be equal $\nu_{+}=\nu_{-} \equiv \nu$ and

$$
\mu_{+}=-\frac{\nu}{2 \pi} \int_{0}^{2 \pi} \frac{d \phi}{f(\phi)}, \quad \nu \in \mathbf{Z} .
$$

Thus two integers $n$ and $\nu$ determine the solution expect a constant $K_{ \pm}(0)$ which can be absorbed to four dimensional spinors $\psi_{ \pm}$. If we, moreover, define a matrix function

$$
H_{+}^{n \nu}=\frac{1}{\sqrt{V_{2}}}\left(\frac{f(0)}{f(\phi)}\right)^{1 / 2} e^{i \nu \theta+i n \phi} e^{a \int_{0}^{\phi} d \phi\left(\mu_{+}+\frac{\nu}{f(\phi)}\right) \gamma^{5}}
$$

the solution of general linear equation (15), the field $N_{+}$can be written as a generalized Fourier expansion

$$
N_{+}=\sum_{n, \nu} H_{+}^{n \nu} \psi^{n \nu}
$$

where $\psi^{n \nu}=\psi_{+}^{n \nu}+\psi_{-}^{n \nu}$ are the corresponding Fourier coefficients of each mode $n, \nu$, i.e. the four dimensional fermion fields.

\section{Effective four dimensional singlet fermions}

The next task is to calculate the spectrum of fermions of the four dimensional effective model. Indeed there will be a double tower of exited states $\psi^{n \nu}$ when the extra dimensions are integrated out. These modes do mix with each other, because the functions $H_{+}^{ \pm}$are not orthogonal in the space of extra dimensions. Thus there appear complicated mixings from both kinetic and mass terms, which should, in principle, be diagonalized.

We consider chiral $\left(M=0, N_{-}=0\right)$ version of Eq. (11):

$$
S_{N}=\int d^{4} x d^{2} z V\left[\frac{i}{2} \bar{N}_{+} \Gamma^{A} \mathcal{D}_{A} N_{+}-\frac{i}{2} \mathcal{D}_{A} \bar{N}_{+} \Gamma^{A} N_{+}\right]
$$

which now can be cast in the form involving four dimensional spinors,

$$
S_{N}=\int d^{4} x d \phi d \theta a f(\phi)\left[i \bar{N}_{+} \gamma^{\mu} \partial_{\mu} N_{+}-\frac{1}{2} \bar{N}_{+} M_{+} N_{+}-\frac{1}{2} \overline{\left(M_{+} N_{+}\right)} N_{+}\right],
$$


where all indices are suppressed. By inserting the expansion (24) into preceding equation we are able to integrate over the extra angular coordinates, i.e. write the action in the form of pure four dimensional theory. However, this four-dimensional action is quite complicated, because neither potential nor kinetic term are diagonal with respect the index $n$. One obtain the action for four-dimensional spinors

$$
\begin{aligned}
S=\int d^{4} x & \left\{\sum_{n n^{\prime} \mu}\left[i A_{n n^{\prime} \nu}^{+} \bar{\psi}_{+}^{n \nu} \not \partial \psi_{+}^{n^{\prime} \nu}+i A_{n n^{\prime} \nu}^{-} \bar{\psi}_{-}^{n \nu} \not \partial \psi_{-}^{n^{\prime} \nu}\right]\right. \\
& \left.-\sum_{n \nu} B \bar{m} \bar{\psi}_{-}^{n \nu} \psi_{+}^{n \nu}+\text { h.c. }\right\}
\end{aligned}
$$

where $\bar{m}$ stands for $\bar{m}=m_{+}+i \mu_{+}$using Eqs. (20) and (22) and, finally, the coefficients of the various terms are

$$
\begin{aligned}
A_{n n^{\prime} \nu}^{+} & =\frac{2 \pi a f(0)}{V_{2}} \int_{0}^{2 \pi} d \phi e^{-i a \Delta m \phi} e^{+2 a \int_{0}^{\phi}\left(\mu_{+}+\frac{\nu}{f(u)}\right) d u}, \\
A_{n n^{\prime} \nu}^{-} & =\frac{2 \pi a f(0)}{V_{2}} \int_{0}^{2 \pi} d \phi e^{-i a \Delta m \phi} e^{-2 a \int_{0}^{\phi}\left(\mu_{+}+\frac{\nu}{f(u)}\right) d u} \\
B & =\frac{4 \pi^{2} a f(0)}{V_{2}}
\end{aligned}
$$

where $\Delta m=\left(n-n^{\prime}\right) / a$. Note, that $B$ is independent on all indices.

Thus we have obtained a infinite double tower of fermionic excitations where kinetic mixings are present. However, at this base of functions the mass terms are diagonal. Of course, if $f$ is a constant function, as on flat torus, all non-diagonal terms vanish, because $\mu_{+}+\frac{\nu}{f} \equiv 0$, and modes decouple from each other.

To proceed, one needs a viable approximation which simplifies the mixed up situation. First, we assume, that there is only two branes. Then a $\theta$-independent scale function defined on the interval $\phi \in[0,2 \pi)$ is now

$$
f(\phi)=H\left(\phi^{\prime}-\phi\right)\left(f_{1}+\Delta f \frac{\phi}{\phi^{\prime}}\right)+H\left(\phi-\phi^{\prime}\right)\left(f_{1}+\Delta f \frac{2 \pi-\phi}{2 \pi-\phi^{\prime}}\right),
$$

where $\Delta f$ and $\phi^{\prime}$ are as previously. The jump condition and the volume are given by Eqs. (17) and (8), respectively. From Eq. (22) one reads now

$$
\mu_{+}=-\frac{\nu}{\Delta f} \ln \frac{f_{2}}{f_{1}}=-\frac{\nu}{f}
$$

where thus $\bar{f}^{-1}=\frac{1}{\Delta f} \ln \frac{f_{2}}{f_{1}}$ is a kind effective size of the $\phi$-direction of the torus.

Suppose now, that $f_{2} \gg f_{1}$, we find that the volume of extra dimensions is essentially $V_{2}=2 \pi^{2} a f_{2}$ and the six-dimensional gravity scale reads $M_{*}^{4}=M_{P l}^{2} /\left(2 \pi^{2} a f_{2}\right)$. We then take into account the physical requirement, that $M_{*} \gtrsim 10 \mathrm{TeV}$. Also, because we really have now a 
four brane, where usual non gauge-singlet particles are bounded with one compact dimension of radius $a$, the massive Kaluza-Klein modes are to be pushed beyond observational limit. The lightest Kaluza-Klein mode has a mass of $2 \pi / a$ which thus has to be high enough, say $2 \pi / a \gtrsim 10 \mathrm{TeV}$, too, i.e. $a \lesssim 0.6 \mathrm{TeV}^{-1}$. Taking the upper limit for $a$ and lower limit for $M_{*}$, and combining these two physical conditions one finds, that $f_{2}$ is large, $f_{2} \sim$ $1.2 \times 10^{24} \mathrm{GeV}^{-1}=2.3 \times 10^{8} \mathrm{~m}$. Inserting these values to Eqs. (28), we find that the ratios $A_{n n^{\prime} \nu}^{ \pm} / A_{n n \nu}^{ \pm} \sim 10^{-16}\left(\phi^{\prime} \sim \pi, n^{\prime} \neq n\right)$ and $A_{n n \nu}^{ \pm}$in practise independent on $\nu$ with $A_{n n \nu}^{ \pm}=B$. Thus, for practical purposes the four-dimensional effective spinor action (27) is diagonal.

We may once more write the spinor action using assumptions given above. The action reads

$$
S=\int d^{4} x\left\{\sum_{n \mu}\left[i \bar{\psi}_{+}^{n \nu} \not \partial \psi_{+}^{n \nu}+i \bar{\psi}_{-}^{n \nu} \not \partial \psi_{-}^{n \nu}-\bar{m}_{n \nu} \bar{\psi}_{-}^{n \nu} \psi_{+}^{n \nu}+\text { h.c. }\right]\right\}
$$

where we have scaled fields as $\psi_{ \pm}^{n \nu} \rightarrow \psi_{ \pm}^{n \nu} / \sqrt{B}$ and made to each mode a suitable chiral rotation in purpose to remove complex mass. The mass $\bar{m}_{n \nu}$ reads now

$$
\bar{m}_{n \nu}=\sqrt{\left(\frac{n}{a}\right)^{2}+\left(\frac{\nu}{\bar{f}}\right)^{2}}
$$

Thus $N \neq 0$ excitations are readily heavy and above direct observational limits. When one requires that also $n=0, \nu \neq 0$ excitations are not directly observable, one has to require $\bar{f}$ to be small enough. Because $\bar{f} \simeq f_{2} / \ln \frac{f_{2}}{f_{1}}$, where $f_{2} \sim 10^{24} \mathrm{GeV}^{-1}$, one has to push $f_{1}$ very low, nearly zero. Indeed one sees, that $\phi=0$ brane lies almost at a gravitational singularity. The geometry of this torus can be compared to real doughnut submerged to $\mathbb{R}^{3}$ : The perimeter of the inner ring is smaller than the perimeter of the outermost ring while at the perpendicular direction the perimeter is independent of the position.

\section{Conclusions and discussion}

In the present paper a six-dimensional low energy model hes been presented. The model contains four-branes and empty bulk. It appears, that in a two four-brane case the requirement of the usual four-dimensional Einstein gravity and non-existence of unwanted light KaluzaKlein modes constraint the model so, that the various modes of gauge-singlet fermion fields decouple. The mass spectrum of these modes, given by (32), contains only heavy effective fermions (besides the zero mode), if the effective size of both directions of the torus are small enough. In the present model it is possible to same time have large Kaluza-Klein masses and keep the volume of the torus large enough, i.e. pull the gravitational scale $M_{*}$ down near 
to its experimantal limit. Thus for each gauge-singlet fermion field there is only one light fermionic mode which couples to ordinary matter, e.g. to left-handed neutrinos. Indeed, mixing with ordinary left-handed neutrinos on the brane(s) results hierarchy of neutrino masses and mixings as studied by several authors.

The model has, of course, many open questions, including the stability of the extra dimensions, the mass spectrum of graviton excitations and the corrections to Newton's law, the neutrino masses and mixing on the brane. In the present paper, we have introduced only ideas, how viable models can be possibly constructed on a curved, compact manifold. More work is certainly needed to construct fully realistic model.

\section{References}

[1] G. Nordström, Phys. Z. 15 (1914) 504; T. Kaluza, Sitzungsber. Preuss. Akad. Wiss. Math. Phys. Kl. (1921) 966; O. Klein, Phys. Z. 37 (1926) 895.

[2] For early considerations towards large extra dimensions, see: I. Antoniadis, Phys. Lett. B 246 (1990) 377.

[3] J. C. Long, H. W. Chan and J. C. Price, Nucl. Phys. B 539 (1999) 23.

[4] J. C. Long, A. B. Churnside and J. C. Price, hep-ph/0009062 C. D. Hoyle et al., Phys. Rev. Lett. 86 (2001) 1418.

[5] For a review, see: E.G. Adelberg et al., Ann. Rev. Nucl. Part. Sci. 53 (2003) 77.

[6] N. Arkani-Hamed, S. Dimopoulos and G. Dvali, Phys. Lett. B 429 (1998) 263.

[7] I. Antoniadis, N. Arkani-Hamed, S. Dimopoulos and G. Dvali, Phys. Lett. B 436 (1998) 257; See also: D. Cremades, L.E.Ibanez and F.Marchesano, Nucl. Phys. B 643 (2002) 93 and C. Kokorelis, Nucl. Phys. B677 (2004) 115.

[8] N. Arkani-Hamed, S. Dimopoulos and G. Dvali, Phys. Rev. D 59 (1999) 086004.

[9] For a review, see e.g. V. A. Rubakov, Phys. Usp. 44 (2001) 871-893.

[10] N. Arkani-Hamed, S. Dimopoulos, G. Dvali and J. March-Russell, Phys. Rev. D65 (2002) 024032 .

[11] K. R. Dienes, E. Dudas and T. Gherghetta, Nucl. Phys. B 557 (1999) 25. 
[12] A. E. Faraggi and M. Pospelov, Phys. Lett. B 458 (1999) 237; G. Dvali and A. Yu. Smirnov, Nucl. Phys. B 563 (1999) 63; A. Das and O. C. W. Kong, Phys. Lett. B 470 (1999) 149; R. N. Mohapatra, S. Nandi and A. Perez-Lorenzana, Phys. Lett. B 466 (1999) 115; R. N. Mohapatra and A. Perez-Lorenzana, Nucl. Phys. B 576 (2000) 466; G. C. McLaughlin and J. N. Ng, Phys. Lett. B 470 (1999) 157; A. Ioannisian and A. Pilaftsis, Phys. Rev. D 62 (2000) 066001; R. Barbieri, P. Creminelli and A. Strumia, Nucl. Phys. B 585 (2000) 28; A. Lukas, P. Ramond, A. Romanino and G. G. Ross, Phys. Lett. B 495 (2000) 136; A. Ioannisian and J. W. F. Valle, Phys. Rev. D 63 (2001) 073002; R. N. Mohapatra and A. Perez-Lorenzana, Nucl. Phys. B 593 (2001) 451; K. R. Dienes and I. Sarcevic, Phys. Lett. B 500 (2001) 133; K. Agashe and G.-H. Wu, Phys. Lett. B 498 (2001) 230; N. Cosme et al., Phys. Rev. D 63 (2001) 113018; D. O. Caldwell, R. N. Mohapatra and S. J. Yellin, Phys. Rev. Lett. 87 (2001) 041601; D. O. Caldwell, R. N. Mohapatra and S. J. Yellin, Phys. Rev. D 64 (2001) 073001; A. S. Dighe and A. S. Joshipura, Phys. Rev. D 64 (2001) 073012; C. S. Lam and J. N. Ng, Phys. Rev. D 64 (2001) 113006; A. Lukas, P. Ramond, A. Romanino and G. G. Ross, JHEP 04 (2001) 010; J.-M. Frere, M. V. Libanov and S. V. Troitsky, JHEP 11 (2001) 025; C. S. Lam, Phys. Rev. D 65 (2002) 053009; A. de Gouvea, G. F. Giudice, A. Strumia and K. Tobe, Nucl. Phys. B 623 (2002) 395; R. Mohapatra, A. Perez-Lorenzana, Phys. Rev. D67 (2003) 075105.

[13] J. Maalampi, V. Sipiläinen, I. Vilja, Phys. Lett. B512 (2001) 91 ; J. Maalampi, V. Sipiläinen, I. Vilja, Phys. Rev. D67 (2003) 113005 .

[14] T. Ghiarghetta and M. Shaposhnikov, Phys. Rev. Lett. 85 (2000) 240; P. Kanti, R. Madden and K. Olive, Phys. Rev. D64 (2001) 044021; I. Kogan et al., Phys. Rev. D64 (2001) 124014; Z. Chagko and A. Nelson, Phys. Rev. D62 (2000); T. Multamäki and I. Vilja, Phys. Lett. B535 (2002) 170; T. Multamäki and I. Vilja, Phys. Lett. B559 (2003) 1 .

[15] L. Randall, and R. Sundrum, Phys. Rev. Lett. 83 (1999) 3370; L. Randall, and R. Sundrum, Phys. Rev. Lett. 83 (1999) 4690. For recent discussion about neutrinos in RS-moden see also: G. Moreau, hep-ph/0407177. 